\title{
Dynamic and static postural alterations in HIV-related progressive multifocal leukoencephalopathy in a Latino-Hispanic male: a case study
}

\author{
Martin G. Rosario, Leah Jamison \\ Texas Woman's University, School of Physical Therapy, Dallas, Texas, United States
}

\begin{abstract}
Introduction: Human immunodeficiency virus (HIV) affects millions of people worldwide. Because of an impaired immune system, HIV-positive people are at a higher risk of contracting HIV-related complications, including progressive multifocal leukoencephalopathy (PML). PML is a progressive disease involving degeneration of the white matter in the central nervous system, causing profound adverse effects on gait and posture. This case report presents gait and posture deficiencies in a physically active 52-year-old Latino-Hispanic male with HIV-related PML.

Case description: System examinations were conducted to evaluate confidence in balance, balance deficits, and fall risk, given the clinical presentation of HIV-related PML. The patient presented a profound balance and gait motor control impairments, with alterations in posture stability, particularly in mediolateral sway, and velocity were increased when adding vestibular input challenge and dual-tasks (counting backward) components. During gait assessment, the patient compensated by reducing speed during the turn and walk back to the sitting position.

Conclusions: This case study strives to provide specific evidence for static and dynamic motor control in PML HIV-related case. Strengthening exercises focusing on posture musculature are highly recommended. Additionally, we encourage to focus on dynamic balance interventions to improve PML-related alterations such the ones presented in this case report.
\end{abstract}

HIV AIDS Rev 2020; 19, 2: 139-145 DOI: https://doi.org/10.5114/hivar.2020.96326

Key words: HIV, balance, gait, motor control, PML.

\section{Introduction}

Human immunodeficiency virus (HIV) affects 36.9 million people worldwide, with 1.1 million living in the United States (US) only. Within the US, Texas and Puerto Rico are among the places with the highest incidence rate, which have increased in the last few years [1]. HIV reproduces within the brain's immune cells, leading to significant cognitive and motor disturbances. These disturbances interfere with everyday activities and are associated with lower quality of life, thus increasing demand for caregivers and healthcare system. Despite advances in antiretroviral therapy (ART), HIV still adversely affects gait and balance, leading to an increased risk for falls, injury, and premature mortality [2-4].
Address for correspondence: Martin G. Rosario, Texas Woman's University, School of Physical Therapy, 5500 Southwestern Medical Avenue, Dallas, TX 75235 United States, e-mail: mrosario1@twu.edu
Article history:

Received: 14.03.2020

Received in revised form: 24.03 .2020

Accepted: 25.03.2020

Available online: 19.06 .2020
International Journal of HIV-Related Problems

HIV \& AIDS

R e v i e w 
Due to the impaired immune system, those with HIV and other diagnoses affecting the immune system are at a greater risk of contracting HIV-related complications such as progressive multifocal leukoencephalopathy (PML) $[5,6]$. PML is a progressive disease involving degeneration of the myelin sheaths in the central nervous system and association of oligodendrocytes through JC papovavirus $[5,6]$ with profound adverse effects on gait and posture. Those with HIV and PML present a higher mortality rate of $30-50 \%$ in the first few months following diagnosis; however, with ART, patients can live longer but suffer neurologic deficits [5, 6]. Among others, some of the PML-related complications are alterations in gait, balance, coordination, speech, and language difficulties [7].

A previous research described the effect of therapeutic training in patients with PML, which focused on helping patients to return to living at home and ambulating inde-

Table 1. Patient's characteristics

\begin{tabular}{l|c}
\hline Characteristics & Values \\
\hline Sex & Male \\
\hline Age & 52 year-old \\
\hline CD4 count & 1.357 \\
\hline BMI & 27.4 \\
\hline Five-time-sit-to-stand test & $11.07 \mathrm{sec}$ \\
\hline Diagnosed HIV & 2002 \\
\hline ABC & 12. average score \\
\hline
\end{tabular}

pendently with an assistive device, after completing an extensive (7-8 months) rehabilitation program [8]. Studies involving postural motor control assessment, especially in Hispanic-Latino population are limited. Therefore, the purpose of this case study was to evaluate dynamic and static postural alterations as well as functional status of a LatinoHispanic individual with HIV-related PML.

\section{Case description}

The purpose of this case report was to present gait and posture deficiencies in a Latino-Hispanic PML HIV-related subject. PML affects areas of the central nervous system, which are essential for balance and coordination, such as the cerebellum. Many of PML-related cases are presented with a general view of impairment with a poor prognosis. In case of our patient, the doctors sent him back home to live the rest of his "few days" comfortably at home with "no chance of recovering". Several studies explained the negative profound effect on balance, coordination, speech, and in turn, quality of life. The current report shows a more detailed approach to perceive balance and to measure dynamic (gait) and static (standing) balance.

\section{History and interview}

A 52-year-old Latino-Hispanic male was referred to physical therapy following a diagnosis of HIV-related PML. Table 1 shows several characteristics of the patient. He was

Table 2. The International Classification of Functioning, Disability and Health (ICF) classification of the patient

\begin{tabular}{|c|c|c|c|}
\hline \multicolumn{4}{|c|}{$\begin{array}{l}\text { Medical diagnosis: HIV-related progressive multifocal leukoencephalopathy (PML) } \\
\text { Physical therapy diagnosis: impaired motor function and sensory integrity associated with progressive disorders of central } \\
\text { nervous system [13] }\end{array}$} \\
\hline Body functions and structures & \multicolumn{2}{|c|}{ Activity limitations } & Participation restrictions \\
\hline $\begin{array}{l}\text { Impaired gait characteristic of cerebellar } \\
\text { ataxia } \\
\text { Impaired vision and depth perception } \\
\text { Impaired muscle coordination } \\
\text { Impaired sensory integration } \\
\text { with balance } \\
\text { Impaired balance } \\
\text { Progressive loss of function secondary } \\
\text { to nature of disease } \\
\text { Aphasia }\end{array}$ & \multicolumn{2}{|c|}{$\begin{array}{l}\text { Inability to ambulate at home } \\
\text { and commune independently } \\
\text { Inability to perform all activities } \\
\text { of daily living }\end{array}$} & $\begin{array}{l}\text { Inability to perform home management } \\
\text { Inability to work as a manager and } \\
\text { bartender at a restaurant } \\
\text { Inability to participate in social activities }\end{array}$ \\
\hline \multicolumn{4}{|c|}{ Contextual factors } \\
\hline \multicolumn{2}{|c|}{$\begin{array}{l}\text { Environmental factors } \\
\text { Lack of transportation } \\
\text { tive duties as a manager and bartender } \\
\text { alth center within home close proximity } \\
\text { SSH apartment on first level } \\
\text { Wheelchair/walker accessible home }\end{array}$} & $\begin{array}{r}\text { Good un } \\
\text { Good } \mathrm{f} \\
\mathrm{I}\end{array}$ & $\begin{array}{l}\text { Personal factors } \\
\text { Motivated } \\
\text { anding of diagnosis and prognosis } \\
\text { and community support system } \\
\text { No comorbidities } \\
\text { mpanying diagnosis of HIV } \\
\text { rase inhibitor drug therapy } \\
\text { Age: } 52 \\
\text { Gender: male }\end{array}$ \\
\hline
\end{tabular}


living independently in San Juan, Puerto Rico, working as a manager of restaurant and a bartender. The patient reported on being HIV-diagnosed in 2002. Before being diagnosed with PML in 2010, he reported "feeling different" and that he had troubles doing everything within his daily routine, particularly involving coordination. Following hospitalization, he was a wheelchair user. The patient was referred to physical therapy in 2011, where he was modified and capable of independent ambulation with a quad cane. In 2017, his abilities to ambulate digressed and he was using a rollator.

The examination strategy formulated the patient's goals, and functional impairments was gathered through a systematic review. The patient reported that he experienced a loss of coordination and balance as well as ataxic gait, leading to his inability to ambulate. Additionally, he expressed that one of his primary goals was to ambulate without assistive devices, which was taken into consideration. The treatment focused on leading causes of his impairments and what could be successfully achieved with a suitable physical therapy.

Table 2 shows a summary of the patient's history and an interview using the International Classification of Functioning, Disability and Health (ICF) classification. The ICF provides insight into the function and abilities in daily life as well as analysis of impairments affecting activity limitation and participation restrictions, thus guiding appropriate and goaloriented interventions for an individualized plan of care [9].

\section{Systems review}

A system review was conducted to assess confidence in balance, balance deficits, and fall risk, given the clinical presentation of HIV-related PML. Upon examination, it was confirmed that the patient has no underlying comorbidities, except for immunocompromise from HIV. He reported that he is presently taking a combination of tivicay and desovy, an integrase inhibitor regimen, for HIV management [10]. Diagnostic techniques used to diagnose PML through MRI and CT scan of the brain demonstrated localized lesions in the affected areas, with insignificant cerebrospinal fluid (CSF) findings [6]. Updated MRI images obtained from 2018 showed demyelination in the corpus callosum (green arrows) and cerebellum (red arrows) as well as atrophy of the cerebellum and cerebral hemispheres (blue arrows) (Figure 1).

Lesions of the corpus callosum can involve an integration and transfer of information from both cerebral hemispheres, thus affecting sensory, motor, and high-level cognitive signals processing [11]. During the development, the corpus callosum seems to represent a crucial role in refining motor movements and cognitive function. A research showed that the corpus callosum has an inhibitory effect that usually prevents uncoordinated hand motor behavior [11]. Lesions of the cerebellum can result in impairments in the coordination of movements, control of posture and gait, and regulation of muscle tone [6]. Given those mentioned above, the clinician determined to assess balance, risk of falls, functional mobility, and gait analysis.

\section{Evaluation}

From the ICF assessment (Table 2), it was evident that the independence in ambulation and daily activities were especially important to the patient. Upon further inspection through the examination and interview process, great difficulty associated with postural stability and dynamic bal-
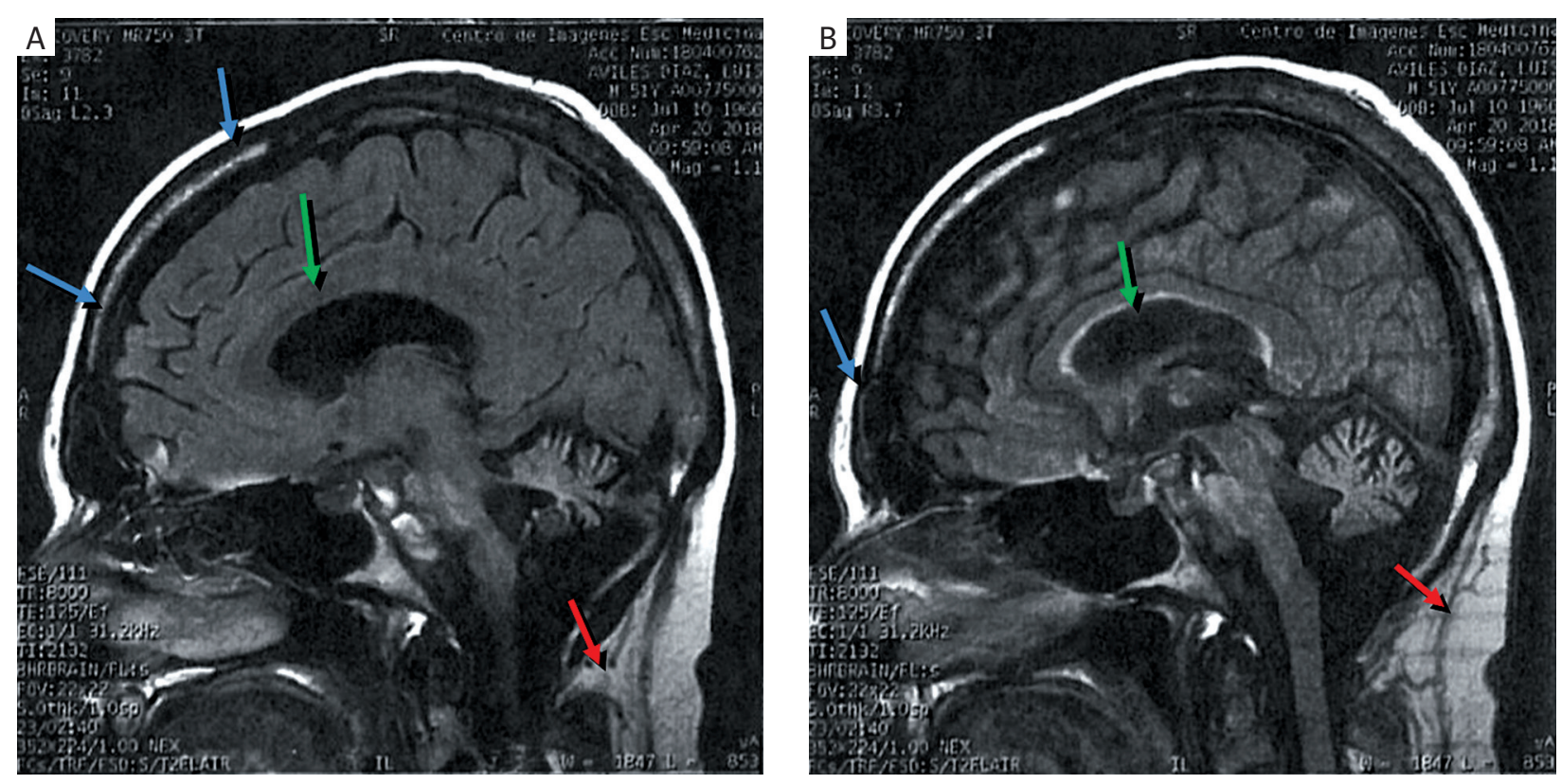

Figure 1. Magnetic resonance imaging from 2018 demonstrating involvement of corpus callosum and cerebellum with cerebellar and cerebellum atrophy. Areas of atrophy are indicated with red arrows 
ance were noted. It is well known that stability and dynamic balance are vital components of locomotor control in maintaining forward propulsion [12]. In a study, Morton et al. evaluated relative contributions of balance versus leg-coordination deficits to cerebellar gait ataxia and demonstrated that those with cerebellar damage with minimal to no balance deficits typically have fewer walking abnormalities than those with significant balance deficits $[12,13]$.

Deterioration in upper and lower extremity coordination was demonstrated during an examination of balance and ambulation, which is characteristic for an individual with cerebellar involvement. A study conducted by Ilg et al. [14] assessed the benefits of an intensive coordinate training program on motor performance in people with degenerative cerebellar disease. Each participant underwent 1 hour of training for 4 weeks and then, 1-hour home exercise program was provided to continue the training until 8 weeks. Those who participated in their home program, regularly demonstrated greater improvements than those who did not [14]. Consequently, the plan of care should be focused on improving the patient's balance, postural stability, and coordination to improve independence with functional daily activities and ambulation for improved quality of life.

\section{Perceived balance}

The ABC scale is patient-specific, and is transferable through all levels of care as the time/cost is minimal and the test is easy to administer [15]. The 16-item ABC scale was administered to the patient (Table 3), assessing his confidence in balance for a variety of daily functional activities without using an assistive device from $0 \%$ confidence (no confidence) to $100 \%$ confidence (complete confidence). The score was calculated by taking the average of the 16 item scores, resulting in a final score of $12.5 \%$. Scores of less than $67 \%$ indicate a high-risk for falling, with $84 \%$ of accurately classifying people who fall [15]. The patient rated $0 \%$ of confidence, with most items involving ambulating on uneven surfaces and in distracting environments as well as ambulating on stairs. He rated $50 \%$ on four items, including walking around the house, retrieving objects from shelves at eye level, getting into and out of a car, and stepping on and off an escalator using the rails for support (Table 3). The balance system requires the interplay of the visual, vestibular, and somatosensory systems. We were expecting balance difficulties in the patient due to his cerebellar atrophy (Figure 1). The cerebellum and the vestibular system $(\mathrm{C} / \mathrm{V})$ are involved in coordination, motor control, and balance. Due to damage to the cerebellar structure by $\mathrm{PML}$, the $\mathrm{C} / \mathrm{V}$ interplay are altered affecting gait and posture. Table 3 shows the reduced balance confidence in several daily activities of the patient. The $\mathrm{C} / \mathrm{V}$ alterations were presented in the $\mathrm{ABC}$ scale of the patient. All the activities that required dynamic balance and coordination had a score of zero. In this $\mathrm{ABC}$ scale, zero indicates no confidence of performing these tasks safely without falling. It is important to point out that our patient reported having difficulties performing all the

Table 3. ABC scale characteristics

\begin{tabular}{|c|c|c|}
\hline Item number & Activity & Score \% \\
\hline 1 & Walk around the house & 50 \\
\hline 2 & Walk up or down stairs & 0 \\
\hline 3 & Bend over and pick up an object & 0 \\
\hline 4 & Bend over and pick up a slipper from the front of a closet floor & 0 \\
\hline 5 & Reach for a small can off a shelf at eye level & 50 \\
\hline 6 & Stand on your tiptoes and reach for something above your head & 0 \\
\hline 7 & Stand on a chair and reach for something & 0 \\
\hline 8 & Sweep the floor & 0 \\
\hline 9 & Walk outside the house to a car parked in the driveway & 0 \\
\hline 10 & Get into or out of a car & 50 \\
\hline 11 & Walk across a parking lot to the mall & 0 \\
\hline 12 & Walk up or down a ramp & 0 \\
\hline 13 & Walk in a crowded mall where people rapidly walk past you & 0 \\
\hline 14 & Bumped into people as you walk through the mall & 0 \\
\hline 15 & Step onto or step off an escalator while you are holding onto a railing & 50 \\
\hline 16 & $\begin{array}{l}\text { Step onto or step off an escalator while holding onto parcels such that you cannot hold onto } \\
\text { the railing }\end{array}$ & 0 \\
\hline Total average score & & $12.5 \%$ \\
\hline
\end{tabular}


16 activities in this questionnaire, giving the item in this tool a $50 \%$ as a high value and $0 \%$ to various of them.

\section{Five times sit to stand test}

The five-times-sit-to-stand test (FTSTS) was selected to assess mobility and function as it measures functional lower extremity strength and movement strategies used for transfers and transitional movements. The FTSTS has excellent test-retest reliability $(\mathrm{ICC}=0.994)$ [16]. The FTSTS was completed to assess transitional and functional movements, while evaluating the risk of falls, with a cut-off score of 12 seconds [17]. The patient completed the task in 11.07 seconds, demonstrating a low-risk for falls and functional lower extremity strength with transitional movement without using an assistive device. Another unexpected, yet important factor, is the normal score of the FTSTS test, presenting the patient's muscle weakness as a factor affecting the balance [18]. In the case of the patient, muscle weakness is unrelated to his balance alteration as proven by his normal FTSTS score. LPGP is a fitness center, which focuses on the wellness and rehabilitation of patients such as our patient. A 3-4 days exercise program was prescribed to the patient for 8 years. The protocol consisted of strengthening, flexibility, and cardiovascular exercises programs designed to halt or slow the balance alteration and in turn, reduce the risk of falls.

\section{Measure balance}

Postural sway under varying conditions uses gyroscopes and body-worn accelerometers sensors (APDM Mobility Lab System). The balance tasks were designed to challenge or cancel the visual, proprioceptive, and vestibular input (Table 4). For each task, including the baseline measurement, the participant was instructed to fixate their gaze on an orange square on the wall 10 feet away. Jackson et al. assessed quiet-standing balance under four testing conditions: standard (single task), dual cognitive task, manual dual-task, and a combined cognitive/manual dual-task in fallers and non-fallers subjects. In their study, participants considered as "fallers" demonstrated significant differences in their ML sway range than those considered "non-fallers," demonstrating that dual tasking affects postural sway in those considered at a higher risk for falls. Considering this mentioned above, a balance protocol was as follows: four non-COG tasks were performed on the foam pad. 1. Eyes open (EO FOAM); 2. Eyes closed (EC FOAM); 3. Eyes open while moving head up and down to the cadence of a $60 \mathrm{bpm}$ metronome (EO HUD); 4. Eyes closed while moving head up and down to the cadence of a $60 \mathrm{bpm}$ metronome (EC HUD).

The dual cognitive tasks component was added to the balance protocol by counting backward from 100 by 3 . The four COG tasks were the same as above, but adding the cognitive components (counting backwards) to a total of 9 tasks. Balance tests protocols are designed to challenge the previously mentioned systems related to standing motor control. PML affects higher cognitive centers and everyday activities required for dual tasks, such as talking and reaching. In an uncompromised cognitive system, this dual-task component has no impact on standing balance. Table 4 showed the higher scores of variables (sway, velocity, and distance of movement) of the patient compared to normative. The measured balance results showed the compromised motor control system of the patient, and how these alterations become more obvious as the tasks become more challenging (single tasks versus dual tasks). The balance data shows the evident alteration in motor control, especially during anterior/posterior jerk and mediolateral velocity. ML jerk and direction are related to a compromised central nervous system [18] such as the cerebellum.

\section{Gait assessment}

As expected with cerebellar involvement, a cerebellar ataxic gait pattern was observed during gait analysis via vid-

Table 4. Standing parameters during balance tasks (post-urography results)

\begin{tabular}{l|c|c|c|c|c|c|c}
\hline Tasks & $\begin{array}{c}\text { Sway } \\
\left(\mathrm{m}^{2} / \mathrm{s}^{4}\right)\end{array}$ & $\begin{array}{c}\text { AP jerk } \\
\left(\mathrm{m}^{2} / \mathrm{s}^{5}\right)\end{array}$ & $\begin{array}{c}\text { ML jerk } \\
\left(\mathrm{m}^{2} / \mathrm{s}^{5}\right)\end{array}$ & $\begin{array}{c}\text { AP velocity } \\
(\mathrm{m} / \mathrm{s})\end{array}$ & $\begin{array}{c}\text { ML velocity } \\
(\mathrm{m} / \mathrm{s})\end{array}$ & $\begin{array}{c}\text { AP distance } \\
\left(\mathrm{m} / \mathrm{s}^{2}\right)\end{array}$ & $\begin{array}{c}\text { ML distance } \\
\left(\mathrm{m} / \mathrm{s}^{2}\right)\end{array}$ \\
\hline EO firm & 0.5372 & 20.591 & 102.685 & 0.0732 & 0.1257 & 21.74 & 50.97 \\
\hline EO foam & 3.1913 & 1092.47 & 362.148 & 0.2692 & 0.3324 & 181.47 & 105.19 \\
\hline EC foam & 4.9707 & 1629.73 & 382.893 & 0.1981 & 0.4185 & 22.9 & 110.04 \\
\hline EO HUD & 1.5106 & 235.794 & 323.318 & 0.08 & 0.165 & 78.27 & 95.11 \\
\hline EC HUD & 9.5203 & 616.122 & 145.626 & 0.204 & 1.3652 & 142.35 & 82.11 \\
\hline EO COG & 2.4501 & 1066.42 & 215.213 & 0.1464 & 0.217 & 177.64 & 77.34 \\
\hline EC COG & 3.8598 & 918.947 & 1144.89 & 0.1045 & 0.6821 & 169.72 & 189.06 \\
\hline EO HUD COG & 1.7946 & 416.013 & 366.165 & 0.0688 & 0.1671 & 114.48 & 103.82 \\
\hline EC HUD COG & 6.4559 & 462.231 & 291.91 & 0.052 & 0.3663 & 136.18 & 115.75 \\
\hline Normative values & $0.0235 \pm 0.0 .01$ & $0.525 \pm 0.4$ & $1.9 \pm 1.2$ & $0.13 \pm 0.1$ & $0.03 \pm 0.02$ & $5.43 \pm 1.7$ & $2.9 \pm 0.9$ \\
\hline
\end{tabular}

EO - eyes open, EC - eyes closed, HUD - head up and down, COG - cognitive task counting backwards from 100.

Significant values from normative are highlighted 
Table 5. Dynamic parameters during two-meter walk test (4 trials)

\begin{tabular}{l|c|c|c|c}
\hline Parameter & Normative mean & Standard deviation & Mean & Standard deviation \\
\hline Duration (s) & 8.76 & 2.3 & 29.01 & 1.06 \\
\hline Sit-to-stand - duration (s) & 0.98 & 0.3 & 1.18 & 0.37 \\
\hline Sit-to-stand - lean angle (degrees) & 32.62 & 12.35 & 31.5 & 6.08 \\
\hline Sit-to-stand - $n(\#)$ & 1 & 0 & 1 & 0 \\
\hline Stand-to-sit - duration (s) & 0.77 & 0.18 & 0.7 & 0.17 \\
\hline Stand-to-sit - lean angle (degrees) & 34.62 & 16.01 & 31.12 & 8.81 \\
\hline Stand-to-sit - $n(\#)$ & 1 & 0 & 1 & 0 \\
\hline Turns - angle (degrees) & 182.17 & 6.67 & 89.3 & 9.4 \\
\hline Turns - duration (s) & 1.94 & 0.38 & 1.8 & 0.27 \\
\hline Turns - $n(\#)$ & & & 2 & 0 \\
\hline Turns - turn velocity (degrees/s) & 230.56 & 49.29 & 126.3 & 9.20 \\
\hline
\end{tabular}

Significant values from normative are highlighted

eo taken during a therapy session. A slow gait with short and unequal step lengths that were irregular and inconsistent in timing were observed, along with a noticeable over exaggeration of hip and knee flexion during the swing phase, all of which are characteristics of a person with cerebellar ataxia [19]. The patient also demonstrated a wide BOS with difficulty in controlling lowering of bilateral lower extremities in transition from mid-swing and term-swing to initial contact. Additionally, he demonstrated greater step length of the right leg than the left leg, with decreased weight shift to the left lower extremity during mid-stance and terminal stance as well as minimal to no reciprocal arm swing and coordination with alternate lower extremity, when ambulating without an assistive device. During the dynamic test while walking a two-meter walkway (Table 5), the patient showed an increased time and angulation (instability) during turning. A reduction in speed and angle suggest that the patient compensated his balance difficulties by slowing down and getting the truck more perpendicular to the floor, less angle for a safer turn-walk back to the starting point. However, this strategy that the patient has adapted increased the risk of falls due to extra and shorter steps while turning [18]. This report recommends focusing on more dynamic parts of gait such as turning and transition of heel strike to toe, to increase the single limb support time and reduce the double limb support time for a better balance control and fall risk reduction.

\section{Conclusions}

The purpose of this paper was to present gait and posture deficiencies in a Latino-Hispanic PML HIV-related subject. PML deteriorates regions of the central nervous system, which are crucial to balance and coordination, such as the cerebellum. Many of PML-related cases concentrate on a general view of impairment with a poor prognosis. Collecting and analyzing measurable values during long-term treatments provides evidence that patients with HIV-related
PML have the potential to become functionally independent across continued therapeutic exercises and interventions. This case study strives to provide such data to further contribute to evidence-based practice, with a value-based approach using outcome measures and therapeutic interventions.

\section{Conflict of interest}

The authors declare no conflict of interest with respect to the research, authorship, and/or publication of this article.

\section{References}

1. Estimated HIV incidence and prevalence in the United. Centers for Disease Control and Prevention. Available at: https://www.cdc.gov/ hiv/pdf/library/reports/surveillance/cdc-hiv-surveillance-supplemental-report-vol-24-1.pdf. Published February 2019 (Accessed: 14.10.2019).

2. Erlandson KM, Allshouse AA, Jankowski CM, et al. Comparison of functional status instruments in HIV-infected adults on effective antiretroviral therapy. HIV Clinical Trials 2012; 13: 324-334.

3. Cohen HS, Cox C, Springer G, et al. Prevalence of abnormalities in vestibular function and balance among HIV-seropositive and HIV-seronegative women and men. PLoS One 2012; 7: e38419.

4. Heinze B, Swanepoel DW, Hofmeyr LM. Systematic review of vestibular disorders related to human immunodeficiency virus and acquired immunodeficiency syndrome. J Laryngol Otol 2011; 125: 881-890.

5. Progressive Multifocal Leukoencephalopathy Information Page. (n.d.). Available at: https://www.ninds.nih.gov/Disorders/All-Disorders/Progressive-Multifocal-Leukoencephalopathy-Information-Page\#disorders-r1

6. Ropper AH, Adams RD, Klein JP, Samuels MA, Victor M. Adams and Victors Principles of Neurology. $7^{\text {th }}$ ed. New York: McGraw-Hill Med; 2001.

7. Snopková S, Štourač P, Fašaneková L, Mihalčin M, Havlíčková K, Svačinka R, et al. Progressive multifocal leukoencephalopathy epidemiology, immune response, clinical differences, treatment. Epidemiol Mikrobiol Imunol 2019 Winter; 68: 24-31.

8. Moreh E, Israel S, Korem M, Meiner Z. Rehabilitation outcome of progressive multifocal leukoencephalopathy in HIV-positive patients: a report of two cases. Disabil Rehabil 2017; 39: 1893-1896. 
9. Bemis-Dougherty A. Practice matters: what is the ICF? 2009, February. Available at: http://www.apta.org/PTinMotion/2009/2/Feature/PracticeMatters/ICF/ (Accessed: 15.04.2019).

10. Guide to physical therapist practice. $3^{\text {rd }}$ ed. Alexandria, VA: American Physical Therapy Association; 2017.

11. Goldstein A, Mesfin FB. Neuroanatomy, Corpus Callosum. [Updated 2018 Oct 27]. In: StatPearls [Internet]. Treasure Island (FL): StatPearls Publishing; 2019. Available at: https://www.ncbi.nlm. nih.gov/books/NBK448209/

12. Morton SM, Bastian AJ. Relative contributions of balance and voluntary leg-coordination deficits to cerebellar gait ataxia. J Neurophysiol 2003; 89: 1844-1856.

13. Umphred DA. Umphred's Neurological Rehabilitation. St. Louis, MO: Elsevier/Mosby; 2013.

14. Ilg W, Synofzik M, Brotz D, Burkard S, Giese MA, Schols L. Intensive coordinative training improves motor performance in degenerative cerebellar disease. Neurology 2009; 73: 1823-1830.

15. Activities-Specific Balance Confidence Scale. 2013, March. Retrieved at: https://www.sralab.org/rehabilitation-measures/activities-specificbalance-confidence-scale

16. Five Times Sit to Stand Test. 2013, June 20. Available at: https://www. sralab.org/rehabilitation-measures/five-times-sit-stand-test\#stroke (Accessed: 15.04.2019).

17. Kenney WL, Lawrence A. ACSMs guidelines for exercise testing and prescription. Baltimore (Md.): Lippincott Williams \& Wilkins; 2010.

18. Shumway-Cook A, Woollacott M. Normal postural control. In Motor Control Translating Research into Clinical Practice. $4^{\text {th }}$ ed. Baltimore, MD: Lippincott Williams \& Wilkins; 2012, 162-166.

19. Umphred DA. Umphred's Neurological Rehabilitation. St. Louis, MO: Elsevier/Mosby; 2013. 